\title{
DE TEXAS, LA GUERRA CONTRA ESTADOS UNIDOS Y OTRAS DESILUSIONES: VISIONES HISTORIOGRÁFICAS DE "LOS AÑOS OLVIDADOS"*
}

\author{
Catherine Andrews \\ Centro de Investigación y Docencia Económicas
}

Para la Dra. Josefina Zoraida Vázquez en agradecimiento y admiración

\begin{abstract}
Dara atender la encomienda de escribir un ensayo historiográfico sobre los artículos publicados en Historia Mexicana que analizan algún aspecto del periodo de 1836 a 1857, o bien desde la promulgación de las Siete Leyes a la Guerra de la Reforma, me di a la tarea de leer cada uno de los 137 textos del acervo de la revista que caen en esta categoría de uno u otro modo. La mayor parte de ellos discuten una periodización más amplia: de todo el siglo XIX, por ejemplo, o de los últimos años de la colonia a la mitad del siglo XIX. Unos 56 artículos se ubican estrictamente dentro del periodo de análisis que me fue asignado por el director de Historia Mexicana, Rafael Rojas. En este breve ensayo, voy a concentrar mi enfoque precisamente en este subgrupo; no obstante, me gustaría hacer algunos comentarios breves sobre el corpus in extenso para contextualizar mis argumentos.
\end{abstract}

* Agradezco a Renata Bautista Mendoza por su apoyo en la recopilación y análisis de los artículos estudiados en este texto. 
En primer lugar, unas observaciones muy formales sobre la estructura de los artículos. Como señalaron Josefina Zoraida Vázquez en ocasión del número 100 de la revista en $1976,{ }^{1}$ y Manuel Miño Grijalva en la conmemoración de su cuadragésimo aniversario en $1991,{ }^{2}$ el estudio de los artículos históricos permite ver la profesionalización de la disciplina de la historia en México. De modo que la extensión promedio de los artículos de mi selección amplia durante la década de 1950 era de 21.3 páginas. A lo largo de sus 70 años de vida, la extensión de los artículos ha crecido paulatinamente: en la última década la extensión promedio de mi muestra amplia es de 48.5 páginas, y se advierten varios textos de más de 70 cuartillas. Es un detalle excepcional entre las revistas académicas regulares que establecen límites muy estrictos en la amplitud de los artículos que reciben. La extensión extraordinaria de los artículos en Historia Mexicana explica la profundidad de análisis que publica regularmente, así como su relevancia e impacto para el trabajo de los historiadores dentro y fuera del país. ${ }^{3}$

Segundo, la revisión de los 137 artículos revela que la profesionalización de la historia en México ha estado acompañada por la gradual diversificación de enfoques y campos de estudio. En ambas selecciones, los artículos sobre la historia política predominan (véase la gráfica 1). La segunda perspectiva más importante -la historia social- tiene una presencia más marcada entre 1970 y 1990; pero desde 2000 está en declive. Al mismo tiempo, la predominancia de la historia política declinó a partir

\footnotetext{
1 VÁzquez, "Historia Mexicana en el banquillo" (100), 1976, pp. 642-654.

2 Miño Grijalva, "Historia Mexicana. Historiografía y conocimiento" (161), 1991, pp. 25-47.

3 En la base de datos de Scimago, Historia Mexicana actualmente cuenta con el puntaje más alto como revista, Q1. Hay pocas revistas en lengua española que llegan a tener este reconocimiento. Véase https://www.scimagojr.com/ journalsearch.php?q=25679\&tip=sid\&clean=0, página consultada el 28 de julio de 2020.
} 
de la década de 1990, en tanto que aparecían más trabajos de historia económica y cultural. En la muestra extendida, se advierte la llegada a las páginas de la revista de la historia conceptual en la década de 2010, pero sólo gracias al número especial 239, coordinado por Guillermo Zermeño en 2011.

\section{Gráfica 1}

ENFOQUES DE LOS ARTÍCULOS DE HISTORIA MEXICANA

PUBLICADOS ENTRE 1950-2010 QUE SE OCUPAN

DEL PERIODO 1836-1856

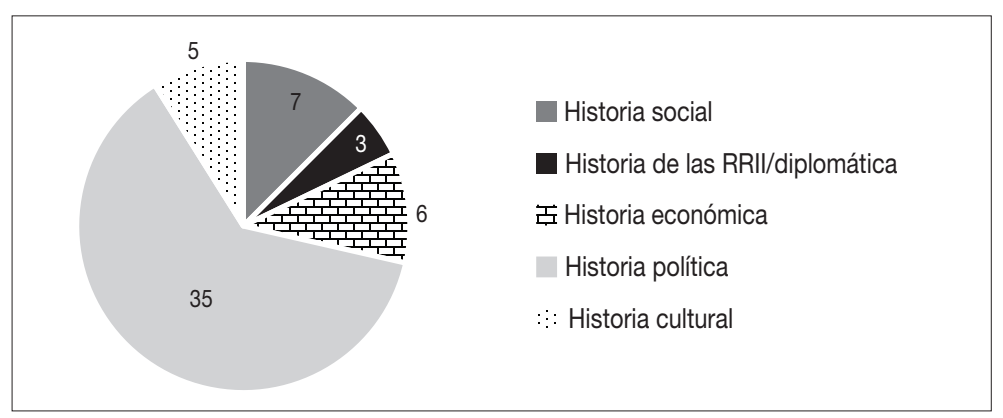

Dentro de la historia política, la guerra contra Estados Unidos es el tema que ha atraído más atención historiográfica, seguido por trabajos sobre Texas, las reformas asociadas con la Revolución de Ayutla y la Iglesia (véase la gráfica 2). Durante la década de 1990, hubo dos números especiales de la revista que reunieron artículos sobre Texas (168) y en torno a la guerra (186). En ambos casos, la coordinación estuvo en manos de Josefina Zoraida Vázquez. Del vol. xLII, provienen tres textos sobre Texas; y del núm. 186, seis sobre la guerra. No obstante, tanto la guerra con Estados Unidos como la historia texana son temas recurrentes en la revista desde su fundación.

En Historia Mexicana, el estudio de la haciendas y cuestión agraria ha sido el más popular de la historia social, seguido por trabajos que estudian los levantamientos campesinos, indígenas 
y populares (véase la gráfica 2). Desde luego, la mayor parte de estos artículos se escribieron entre las décadas de 1970 y 1990, el periodo de máximo interés por la tierra y el trabajo. Se percibe la influencia de Jan Bazant, Robert Knowlton y Moisés González Navarro, tres de los colaboradores más asiduos de la revista durante este periodo, pero también la presencia de autores clave de la historiografía, como Romana Falcón y Florencia Mallon. En la selección amplia, Bazant aparece seis veces, González Navarro cuatro y Knowlton tres.

\section{Gráfica 2}

TEMAS DE LOS ARTÍCULOS DE HISTORIA MEXICANA PUBLICADOS ENTRE 1950-2010 QUE SE OCUPAN DEL PERIODO 1836-1857

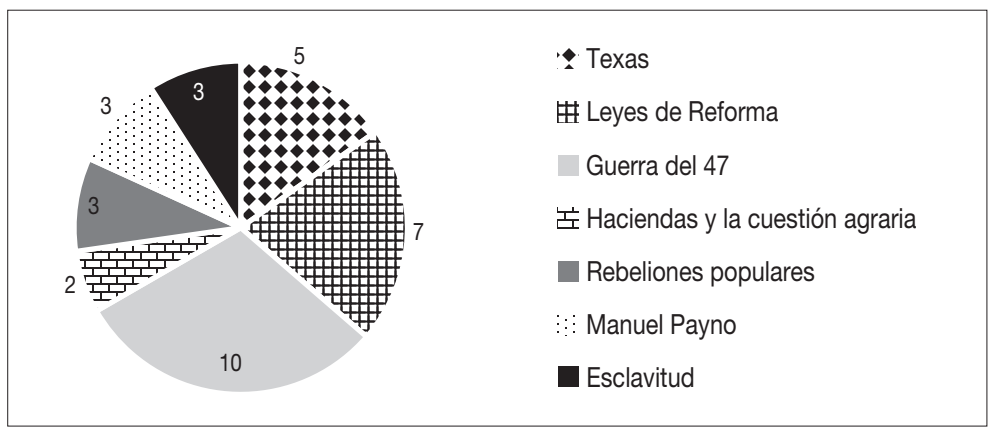

Nadie que revise los artículos de Historia Mexicana que tratan del periodo de 1836 a 1857 puede ignorar la enorme contribución de Josefina Zoraida Vázquez a la revista. Es la investigadora que más textos ha publicado, tanto en el caso de la muestra grande como en la corta. De hecho, escribió 7 de los 57 artículos que cubren exclusivamente el periodo de las Siete Leyes a la Guerra de Reforma; y, como remarqué antes, gracias a su impulso Texas y la Guerra de 1847 dominan la historia política. Vázquez igualmente es autora de uno de los artículos más importantes de la historia política del periodo en cuestión: 
"Iglesia, ejército y centralismo" (1997), ${ }^{4}$ que cuestiona y replantea muchos de los tópicos historiográficos relacionados con el centralismo y sus vínculos con el conservadurismo que defendía los privilegios del ejército y la Iglesia. El ensayo en cuestión forma parte de una serie de artículos que la doctora publicó en Historia Mexicana (y en otras revistas) durante la década de 1990 que demostraron la visión liberal compartida entre federalistas y centralistas durante la primera mitad del siglo xIx, y dieron pie a varias de las nuevas interpretaciones históricas de esta época, incluyendo, hay que decirlo, las mías.

En reconocimiento de los aportes de la doctora Vázquez, tanto a este campo como al estudio de la guerra contra Estados Unidos, decidió estructurar este breve ensayo en torno a los artículos de Historia Mexicana que abordan estas cuestiones. En el siguiente apartado, discutiré los trabajos que han estudiado la guerra, con referencia igualmente a varios textos que examinan la historia temprana de Texas. En seguida, pasaré a discutir las aportaciones de Vázquez a la historia política, para concluir con un breve repaso de algunos de los artículos más importantes para la historia política de lo que llamaba "los años olvidados" del siglo XIX.

\section{TEXAS Y LA GUERRA CONTRA ESTADOS UNIDOS}

Los historiadores de México sabemos que hay un antes y un después de la guerra con Estados Unidos: en las fuentes vemos cómo la invasión de 1847 llevó a la recriminación, la desesperación y un fuerte cuestionamiento sobre la naturaleza misma de la nación mexicana. ¿Cómo podía haber ocurrido semejante tragedia? ¿A quién o a quiénes se podría culpar por la humillación de perder la mitad del territorio nacional a manos de los estadounidenses? Un poco más de 100 años después, estas

${ }^{4}$ VÁzquez, "Iglesia, ejército y centralismo” (153), 1989, pp. 205-234. 
preguntas seguían presentes y siendo relevantes para los primeros colaboradores de Historia Mexicana. Si durante las décadas posteriores a la guerra, la tendencia política fue suponer que las respuestas a estas preguntas se encontraban en la debilidad del patriotismo nacional, para la década de 1950 los historiadores prefirieron poner énfasis en la fiebre anexionista estadounidense y la complicidad traicionera del general Antonio López de Santa Anna con los planes del gobierno del presidente Polk.

Así las cosas, en el número 1 del segundo volumen de la revista (septiembre de 1952), hay dos artículos sobre el tema: "La guerra a México de Estados Unidos a México”, que reproduce el capítulo acerca de la guerra del segundo volumen de Historia de México (1941) de José Bravo Ugarte, ${ }^{5}$ y "Dos diplomacias y un problema” de Carlos Bosch García. ${ }^{6}$ El primero traza el desencuentro diplomático de la década de 1840 y los principales acontecimientos de la guerra, mientras que el segundo estudia el intercambio diplomático entre los representantes de México y Estados Unidos en torno al reconocimiento de la anexión de Texas por parte del vecino norteño. En ambos textos, se señala la duplicidad de los argumentos del gobierno norteamericano frente a México, y sus intentos de presentar la anexión de Texas y la posterior invasión como actos provocados por el mal gobierno mexicano y su implacable (e injusta) hostilidad a los texanos. Dos años más tarde, en el volumen IV: 1, Luis Nicolau d'Owler publicó “Santa Anna y la invasión vistos por Bermúdez de Castro”, en que analiza el papel del caudillo en la guerra a partir de los informes del embajador español, donde el rumor de un acuerdo previo entre Santa Anna y el gobierno de Estados Unidos es ampliamente citado. ${ }^{7}$ 'Owler termina

5 Bravo Ugarte, “La guerra a México de Estados Unidos (1846-1848)” (2), 1951, pp. 185-226.

6 Bosch García, "Dos diplomacias y un problema” (5), 1952, pp. 46-65.

7 D’Olwer, "Santa Anna y la invasión vistos por Bermúdez de Castro” (13), 1954, pp. 47-65. 
su texto preguntándose cómo el historiador debería juzgar a Santa Anna: “'Traidor? Su único ideal era el ejercicio del poder supremo; para lograrlo y mantenerse en él jugó con los ideales ajenos. Federación, democracia, acercamiento a Europa..., sólo fueron naipes de baraja política en las manos de aquel jugador empedernido". 8

Cuarenta años más tarde, en el número conmemorativo de los 150 años de la guerra con Estados Unidos (1997), los colaboradores presentaron un análisis mucho más matizado y equilibrado de las relaciones entre México y Estados Unidos. Dos artículos examinan el contexto político y las actitudes culturales de Estados Unidos frente al expansionismo. Robert M. Johannsen analiza el discurso político que lo motivó. Señala la potencia de la metáfora de "la joven América”, cuya virilidad y vigor comparaba con el estupor de la decadente Europa. En este discurso, la expansión hacia el occidente se veía como una consecuencia inevitable del crecimiento de un país que corría hacia el futuro como un tren sobre el ferrocarril. En este relato, la guerra contra México se hizo en nombre de la modernización y la civilización: las tropas llevarían democracia y libertad a un país subyugado por el peso de la herencia colonial. ${ }^{9}$ Por su parte, Jesús Velasco Márquez ofrece un estudio pormenorizado de las disputas y tensiones alrededor de la incorporación de nuevos estados a la Unión Americana previa a la guerra. Como observa el autor, no toda la población americana compartía el discurso del "Destino Manifiesto"; por consiguiente, la expansión fue motivo de fuertes polémicas entre los partidos políticos y sus seguidores. ${ }^{10}$

8 D’Olwer, “Santa Anna y la invasión vistos por Bermúdez de Castro” (13), 1954, p. 63.

9 Johannsen, “La joven América y la guerra con México” (186), 1997, pp. 261-284.

10 Velasco Márquez, “Regionalismo, partidismo y expansionismo” (186), 1997, pp. 311-343. 
Del lado mexicano, el contexto político y cultural fue abordado por Josefina Zoraida Vázquez, quien examina el intercambio diplomático entre los ministros plenipotenciarios de Estados Unidos y México en los años inmediatamente anteriores a la guerra, ${ }^{11}$ y por Andrés Reséndiz Fuentes, quien estudió el discurso nacionalista mexicano antes de la guerra. ${ }^{12}$ En ambos artículos, el costo psicológico y político de la independencia de Texas se perfila como el factor que determinó la reacción mexicana frente a su posterior anexión a Estados Unidos. El dossier de 1997 también incluye un trabajo fascinante de Robert Ryal Miller en torno a los famosos soldados del Batallón de San Patricio y sus motivos para pasarse al bando mexicano, ${ }^{13}$ así como la publicación del diario que escribió Mariano Riva Palacio durante la invasión. ${ }^{14}$ La presentación de este documento estuvo a cargo de Vázquez y complementó dos tesoros archivísticos más sobre la guerra, que la autora había publicado en Historia Mexicana en 1966 y 1987: la traducción del discurso del diputado Robert MacLane, político que luego firmaría un tratado con el gobierno de Benito Juárez en 1859, en defensa de la guerra, y en favor de la conquista e incorporación de la República mexicana a Estados Unidos, ${ }^{15}$ y la traducción de las comunicaciones entre el ministro británico en México, Charles Bankhead y Lord Palmerston, que dan cuenta de los esfuerzos británicos para convencer al gobierno mexicano de reconocer la independencia de Texas. ${ }^{16}$

11 VÁzQuez, “El origen de la guerra con Estados Unidos” (186), 1997, pp. 285-309.

12 Reséndez Fuentes, “Guerra e identidad nacional” (186), 1997, pp. 411-439.

13 Miller, “Los san patricios en la guerra de 1847” (186), 1997, pp. 345-385.

14 Vázquez, "Breve diario de don Mariano Riva Palacio (agosto de 1847)" (186), 1997, pp. 441-445.

15 VÁzquez, “McLane y la intervención norteamericana” (62), 1966, pp. 274-292.

16 VÁzquez, "Santa Anna y el reconocimiento de Texas” (143), 1987, pp. 553-562. 
La importancia de Texas como factor explicativo de la tragedia de la guerra con Estados Unidos significa que las circunstancias de su independencia también son de mucha consideración para la historia mexicana. Desde el lado texano, se enfatiza que uno de los factores determinantes de esta ocurrencia fue la imposición del centralismo en México y la abolición consecuente de los gobiernos autónomos federales. Esta interpretación incluye la idea de que los angloamericanos hicieron causa común con los federalistas durante la república central. En 1957, Horace Harrison publicó un artículo en Historia Mexicana, por ejemplo, que argumenta que los federalistas de las villas del norte de Tamaulipas y sus aliados, como José Antonio Mejía, buscaban una alianza con el gobierno texano a finales de la década de 1839 para igualmente separarse de México y establecer una república federal independiente. ${ }^{17}$

La presencia de vínculos políticos y sociales entre las poblaciones de Texas y las villas de la frontera encuentra mucho eco en las páginas de la revista. Hay trabajos que estudian los matrimonios, el comercio y el contrabando transfronterizo. ${ }^{18}$ En 1981, Robert Case publicó un texto que detalla la manera en que los diferentes oponentes de los gobiernos mexicanos entre 1850 y 1900 se establecieron en Texas, desde donde lanzaban sus movimientos revolucionarios. ${ }^{19}$ No obstante, y gracias en gran parte al trabajo de Vázquez, la historiografía mexicana rechaza la sugerencia de que Texas se independizó por culpa de México. Un trabajo importante en este sentido es su artículo " $\mathrm{La}$ supuesta República del Río Grande”, en el que demuestra que las

17 Harrison, "Los federalistas mexicanos de 1839-40 y sus tanteos diplomáticos en Texas" (23), 1957, pp. 321-349.

18 Cerutti y González Quiroga, “Guerra y comercio en torno al río Bravo (1855-1867)” (158), 1990, pp. 217-297; GutMann, Hopkins y Fliess, “Matrimonio y migración en la frontera" (197), 2000, pp. 45-76.

19 CASE, "La frontera texana y los movimientos de insurrección en México" (119), 1981, pp. 415-452. 
alianzas entre federalistas mexicanos y texanos durante la vigencia de la Constitución de las Siete Leyes no tenía como objetivo la independencia de las villas del norte. ${ }^{20}$ Ésta fue una invención de los periódicos texanos que interpretaron las declaraciones a favor de promulgar una república federal como un manifiesto en pro de la independencia, en lugar de un pronunciamiento que buscaba derrocar la república central en México para regresar al sistema federal.

En el número especial sobre Texas publicado en 1993 se abordaron otros mitos de la historia texana. Por ejemplo, Andreas Reichstein se preguntó si es correcto pensar que su independencia fue el resultado "inevitable" de un "conflicto de culturas". ${ }^{21}$ Su respuesta es un retundo "no". Señala las buenas relaciones entre los hacendados mexicanos en Texas con sus contrapartes angloamericanos hasta 1835 , y la facilidad con que políticos mexicanos del sur, como Lorenzo de Zavala y José Antonio Mejía, se integraron a la vida económica texana. Asimismo, rechaza la hipótesis de que la rebelión de 1835 ocurrió en contra del "centralismo" del gobierno de Antonio López de Santa Anna y argumenta, en cambio, que la separación de Texas derivó de una pugna entre los especuladores de tierra en Texas y las familias ligadas a Stephen Austin. Según Reichstein, Austin se pronunció por la independencia para intentar unir a los colonos angloamericanos en contra de las grandes compañías de tierras. Reichstein afirma que después de la guerra "un espíritu de venganza" se apoderó de los angloamericanos, llevándolos a perseguir a sus vecinos mexicanos. En un texto del mismo número, Paul Lack retoma este aspecto de la independencia texana, concentrándose en "Los tejanos leales a México" que vivían en la región alrededor de Nacogdoches en la frontera con

20 VÁzquez, “La supuesta República del Río Grande” (141), 1986, pp. 49-80.

21 ReICHSTEIN, “¿Era realmente inevitable?” (168), 1993, pp. 867-887. 
Luisiana. ${ }^{22}$ Lack demuestra que los hacendados y rancheros de origen mexicano fueron hostigados por las nuevas autoridades texanas, despojados de sus tierras y expulsados de Texas por la sospecha de su continuada lealtad a la República mexicana.

EN CONTRA DE LA HISTORIOGRAFÍA TRADICIONAL

"Iglesia, ejército y centralismo" se publicó en 1989 en el número 153 de Historia Mexicana. Le siguió "Un viejo tema: el federalismo y el centralismo" cuatro años después en el número 167. En el mismo periodo, pero en otros espacios, también aparecieron "El fracaso del liberalismo centralista mexicano", "Centralistas, conservadores y monarquistas, 1830-1853" y "Los años olvidados", título que he tomado para nombrar este artículo. ${ }^{23}$ Estos textos plantearon la querella de Josefina Zoraida Vázquez con una historiografía que -todavía en la década de 1990- interpretaba la historia de las décadas de 1830 y 1840 como un conflicto entre centralistas conservadores vs. federalistas liberales. Como señala Vázquez en "Iglesia, ejército y centralismo", el origen de esta exégesis se encontraba "en las acusaciones partidistas contemporáneas a los hechos" y en la interpretación que José María Luis Mora ofreció de la historia patria en "Revista política": como un enfrentamiento entre las fuerzas de progreso y las de orden o retroceso. ${ }^{24}$ Detrás de los partidarios del orden, acechaban los clérigos y los militares: dos corporaciones que quisieron recrear el gobierno colonial mediante la imposición del sistema central con el fin proteger sus históricos privilegios a expensas

22 LACK, "Los tejanos leales a México" (168), 1993, pp. 889-917.

${ }^{23}$ Vázquez, "Los años olvidados”, pp. 313-326. Vázquez, "El fracaso del liberalismo centralista mexicano", pp. 109-121; VÁzQuEz, "Centralistas, conservadores y monarquistas", pp. 115-133.

${ }^{24}$ Mora, "Revista política”, pp. 292-547. 
del bienestar general del pueblo, cuya felicidad dependía del triunfo eventual del federalismo..$^{25}$

El problema principal con esta tesis -dice Vázquez- es que "la revisión de los planes políticos y la folletería contemporánea no parecen sustentar esa supuesta conspiración del clero y la milicia para establecer el centralismo". ${ }^{26}$ De hecho, una revisión cuidadosa de las fuentes ilustra que la coalición política que impulsó el centralismo fue fundamentalmente de orientación liberal y poseía una preocupación auténtica "por el país que parecía en camino de desintegrarse". ${ }^{27}$ En consonancia con la investigación de su alumno, Reynaldo Sordo Cedeño, Vázquez subraya que ni el ejército ni la Iglesia ostentaban una representación desbordada ni significativa en el Congreso Constituyente de 1836. No hubo ningún plan secreto para establecer un gobierno despótico, sino que el trabajo de los diputados "fue honesto y tenía sin duda la finalidad de salvar al país". ${ }^{28}$

En "Un viejo tema”, Vázquez se explayó sobre el punto. La narrativa de pugna externa entre federalistas-liberales $v$ s. conservadores-centralistas olvidaba "que el liberalismo europeo era en general centralista y que las dos corporaciones [iglesia y ejército] distaban de ser monolíticas". ${ }^{29}$ Había militares y clérigos en ambos grupos. Enfatizaba que los políticos mexicanos del periodo temprano independiente fueron altamente influenciados por "el pensamiento gaditano" que consideraba indispensable "un gobierno central fuerte". ${ }^{30} \mathrm{El}$ conflicto entre federalistas y centralistas, por ende, debería entenderse como un enfrentamiento entre los liberales de inclinación gaditana " $\mathrm{y}$ aquellos que defendían el grado de autonomía conquistado por

\footnotetext{
25 VÁzQuez, “Iglesia, ejército y centralismo” (153), 1989, pp. 205-207.

26 VÁzquez, “Iglesia, ejército y centralismo” (153), 1989, p. 205.

27 VÁzQuez, “Iglesia, ejército y centralismo” (153), 1989, p. 230.

28 VÁzquez, “Iglesia, ejército y centralismo” (153), 1989, p. 230.

29 VÁzQuez, “Un viejo tema” (167), 1993, p. 623.

30 VÁzquez, “Un viejo tema” (167), 1993, p. 624.
} 
las élites provinciales a partir de la organización de las intendencias", como Nettie Lee Benson había argumentado desde 1955. A la influencia gaditana se debe atribuir la división de poderes de la Constitución de 1824, en que "[e]l poder legislativo fue el dominante, y además de abusar de sus facultades mantuvo un enfrentamiento constante con el ejecutivo" ${ }^{31} \mathrm{~A}$ las pretensiones de las élites locales se debían los elementos del "antifederalismo jeffersoniano" que adornaban la carta federal, gracias a los cuales el gobierno general gobernaba "estados, no ciudadanos". ${ }^{32}$

Es imposible desestimar la importancia de aquellos trabajos de Josefina Zoraida Vázquez para quienes nos formamos en las décadas de 1990 y 2000. En lo personal, he utilizado sus argumentos en los dos textos que he publicado en Historia Mexicana para insistir en que las Siete Leyes derivaron de las críticas a la división de poderes y a la amplitud poco definida de la soberanía estatal reconocida en la Constitución de $1824 .{ }^{33}$ Lejos de ser una constitución que instaurara el autoritarismo, las Siete Leyes erigieron una arquitectura constitucional diseñada para limitar constitucionalmente al Congreso General y para subordinar las legislaturas de los estados a la autoridad del gobierno nacional. Asimismo, entre los demás artículos de la revista estudiados aquí, también se percibe su influencia en el artículo de Rafael Rojas acerca de la evolución del pensamiento de José María Luis Mora durante su largo exilio en París. ${ }^{34}$ Finalmente, hay que decir que gracias a su impulso, a partir de 2000 hubo un nuevo interés en estudiar el federalismo mexicano: ahora desde la perspectiva de los estados y con base en las fuentes primarias de los archivos locales. A casi 20 años de los artículos de Vázquez en

31 VÁzquez, “Un viejo tema” (167), 1993, p. 624.

32 VÁzQuez, "El fracaso del liberalismo centralista mexicano”, pp. 111-112.

33 Andrews, "Discusiones en torno a la reforma” (221), 2006 pp. 76-116; y Andrews, “El legado de las Siete Leyes” (272), 2019, pp. 1539-1591.

34 Rojas, “Mora en París (1834-1850)” (245), 2012, pp. 7-57. 
Historia Mexicana, conocemos con mucho más profundidad la historia del federalismo en México.

\section{NUEVAS PERSPECTIVAS SOBRE LOS AÑOS NO TAN OLVIDADOS}

Con relación a la historia política de la primera mitad del siglo xix, la producción historiográfica de Historia Mexicana desde 2000 ha aportado diversas perspectivas nuevas. Es evidente que "los años olvidados" ya no merecen este nombre; hay una diversidad de artículos cuya temática sobrepasa el enfoque tradicional del quehacer gubernativo. Es todavía notorio, sin embargo, que el periodo entre 1836 y 1857 sigue sin recibir mucha atención histórica. Para concluir este breve ensayo, quiero repasar unos de los artículos que ofrecen nuevas pistas para entender la primera mitad del siglo XIX en general y señalar de manera particular a los pocos que también se ocupan de los 20 años posteriores a la promulgación de las Siete Leyes.

En primer lugar, hay que remarcar que varios artículos que menciono provienen de los números especializados de la revista publicados desde 2010: el número 239, de 2011, sobre la historia conceptual, y los números 273 y 274 de 2019 que contienen respectivamente un dossier sobre la libertad de la prensa y otro sobre la esclavitud y su abolición en Hispanoamérica. Del número sobre la historia conceptual, destaco los trabajos sobre el significado del concepto "representación” y "razón” entre 1750 y 1850 de Aquiles Omar Ávila Quijas y Priscila Pilatowsky Goñi. ${ }^{35}$ Ambos artículos abren nuevos horizontes sobre la interpretación del discurso político del gobierno independiente. Por otra parte, el texto de Carolina González Undurraga sobre el concepto de raza es una buena lectura complementaria a los

35 Ávila Quijas, "La transición de la Nueva España al México republicano" (239), 2011, pp. 1453-1489; Pilatowsky GoÑI, "Sobre el concepto razón” (239), 2011, pp. 1553-1594. 
publicados en el dossier acerca de la esclavitud y su abolición: sobre todo, el artículo de Celso Castilho acerca de las dinámicas raciales visibles en la presentación de la obra La cabaña del Tío Tom en la ciudad de México y el análisis de Jorge E. Delgadillo Nuñez en torno al uso político del tema de la esclavitud en los periódicos de la ciudad de México entre 1840 y $1860 .^{36}$

El dossier sobre la libertad de prensa presenta dos artículos que caen dentro de los límites establecidos arriba: la investigación de Laurence Coudart sobre la libertad de prensa en las memorias de los ministros de Relaciones entre 1821 y 1860,37 y el texto de Víctor Villavicencio Navarro acerca de los cargos presentados contra José María Gutiérrez de Estrada y del editor Ignacio Cumplido en 1839 por escribir y publicar una carta abierta al presidente Anastasio Bustamante para proponer el establecimiento de una monarquía constitucional en México. ${ }^{38}$ Coudart demuestra con mucha claridad "la función social" que los sucesivos gobiernos esperaban de la prensa periódica como formadora de la opinión pública y el papel político que ocupaba en consecuencia, en tanto que se consideraba el foro principal para difundir los valores nacionales. Este papel permite entender las leyes de imprenta y su relación con la libertad de prensa consagrada en todas las constituciones mexicanas.

El texto de Villavicencio Navarro ofrece un ejemplo muy importante de esta relación al detallar el modo en que la administración de Bustamante procedió judicialmente contra Gutiérrez de Estrada y Cumplido. Se alegó que se trataba de un acto de subversión por atentar contra "la constitución y sus bases", y

36 Castilho, “La cabaña del tío Tom” (274), 2019, pp. 789-835; Delgadillo NúÑEz, "La esclavitud, la abolición y los afrodescendientes” (274), 2019, pp. 743-788.

37 Coudart, “La libertad de imprenta en los informes ministeriales” (273), 2019, pp. 205-256.

38 Villavicencio Navarro, "Cuando la prensa incomoda al sistema político” (273), 2019, pp. 159-204. 
por contravenir la ley del 23 de abril de 1824 en que se establecía que la persona que ayudara a Iturbide a regresar a México, o "de alguna manera protegieran las miras de cualquier invasor extranjero", se consideraría traidora y sería juzgada de acuerdo con el decreto del 27 de septiembre de 1823. Es decir, por tribunales militares. No importaba que aquella ley de 1823 estuviera derogada $3{ }^{39}$ clasificar el folleto en estos términos permitió al presidente alegar que el contenido del folleto era suficientemente perturbador del "bien y la seguridad pública" para merecer un castigo más allá de lo previsto por las leyes de imprenta vigentes.

Finalmente, me gustaría resaltar dos artículos que dan cuenta del modo en que las nuevas perspectivas historiográficas de los últimos treinta años han arrojado luz sobre los temas predilectos de los primeros autores de Historia Mexicana: las Leyes de Reforma. En primer lugar, me refiero al texto de Pablo Mijangos "Entre la igualdad y la gobernabilidad: los motivos de la supresión del fuero eclesiástico". ${ }^{40}$ En este escrito, Mijangos deja de un lado los argumentos que ven la Ley Juárez de 1856 como una manifestación del principio liberal de igualdad, para indagar acerca de "las posibles motivaciones prácticas" detrás del decreto. ${ }^{41}$ Para hacerlo, analiza cuidadosamente la historia de los (des)encuentros entre los fueros civil y eclesiástico durante las décadas inmediatamente anteriores a 1857. De esta manera, muestra cómo las grandes variaciones entre la jurisdicción y la normatividad de los tribunales eclesiásticos y los civiles creaban problemas políticos durante el primer federalismo y, dentro de este contexto, cómo la inmunidad del clero empezaba a interpretarse como garante de impunidad para los clérigos delincuentes

39 La ley del 27 de septiembre de 1823 fue derogada el 18 de diciembre de 1832. Véase Dublán y Lozano (eds.), Legislación mexicana, vol. II, p. 470.

40 Mijangos y González, “Entre la igualdad y la gobernabilidad” (261), 2016, pp. 7-64.

41 Mijangos y González, “Entre la igualdad y la gobernabilidad” (261), 2016, p. 12. 
y una herramienta con que la Iglesia podría entrometerse en asuntos exclusivos del fuero civil.

El segundo artículo es "Pueblos, reformas y desfases en el sur de México: Oaxaca, 1856-1857”, de Luis Alberto Arrioja Díaz Virruell, que examina la recepción y resistencia frente a la Ley Lerdo de 1856 en Oaxaca. ${ }^{42}$ En este texto, Arrioja Díaz Virruell demuestra que el discurso triunfal de la historia política liberal con relación a las Leyes de Reforma requiere matices importantes. Observa que el entusiasmo con que el gobierno oaxaqueño promovió la desamortización de las corporaciones civiles en 1856 no era algo nuevo para los políticos oaxaqueños, sino que reflejaba un interés recurrente por parte de los sucesivos gobernadores de Oaxaca "en disolver la condición jurídica que resguardaba los bienes comunales". ${ }^{43} \mathrm{Al}$ mismo tiempo, compara este contexto con la Constitución oaxaqueña en 1857, sobre todo con el artículo 68 párrafo 4, que otorgaba a los ayuntamientos la facultad "de administrar los bienes comunales", y se pregunta por las razones de esta disyuntiva. ${ }^{44}$ Encuentra la respuesta en la oposición a la desamortización de varios grupos, principalmente políticos conservadores, defensores de la Iglesia y de las propias comunidades afectadas, lo que obligó a los políticos liberales oaxaqueños a buscar una solución negociada con el fin de evitar una confrontación abierta. Arrioja concluye, sobre todo, que "el lugar que ocupaban los pueblos y los bienes comunales indígenas en la economía estatal” fue determinante para frenar el refrendo de la Ley Lerdo en la constitución local. ${ }^{45}$

42 Arrioja Díaz Viruell, "Pueblos, reformas y desfases en el sur de México" (254), 2014, pp. 487-532.

43 Arrioja Díaz Viruell, "Pueblos, reformas y desfases en el sur de México" (254), 2014, p. 493.

44 Arrioja Díaz Viruell, "Pueblos, reformas y desfases en el sur de México" (254), 2014, p. 500.

45 Arrioja Díaz Viruell, "Pueblos, reformas y desfases en el sur de México" (254), 2014, p. 523. 
Los artículos mencionados en este último apartado son testimonio de las aportaciones de Historia Mexicana a la historiografía nacional y la importancia de la revista para las nuevas generaciones de historiadores. Tal vez es un tópico decir que toda historia responde a las inquietudes del presente, pero en el contexto actual es notorio que en las páginas de la revista se puede apreciar que la narración histórica sobre las primeras décadas de independencia ha superado las limitaciones del discurso patriótico liberal que criticó Josefina Zoraida Vázquez en las décadas de 1980 y 1990, para presentar una apreciación diversa de sus complejidades. El reto para los siguientes años es lograr que estas interpretaciones tengan un impacto positivo en el discurso político popular.

\section{REFERENCIAS}

Dublán, Manuel y José María Lozano (eds.), Legislación mexicana o colección completa de las disposiciones legislativas expedidas desde la independencia de la República, México, Imprenta del Comercio, a cargo de Dublán y Lozano, hijos, calle de Cordobanes, número 8, 1876, vol. 2.

Mora, José María Luis, “Revista política”, en Obras Completas de José María Luis Mora, investigación, recopilación y notas de Lilian Briseño Senosiain, Laura Solares Robles y Laura Suárez de la Torre, México, Instituto de Investigaciones Dr. José María Luis Mora, 1986, vol. 2.

VÁzquez, Josefina Zoraida, "Centralistas, conservadores y monarquistas, 1830-1853”, en Humberto Morales y William Fowler (coords.), El conservadurismo mexicano en el siglo XIX (1850-1910), Puebla, Benemérita Universidad Autónoma de Puebla, University of Saint Andrews, Secretaría de Cultura, Gobierno del Estado de Puebla, 1999, pp. 59-85.

VÁzQuEz, Josefina Zoraida, "El fracaso del liberalismo centralista mexicano", en Anuario de IEHS, 11 (1996), pp. 109-21.

VÁzquez, Josefina Zoraida, “Los años olvidados”, en Mexican Studies/Estudios Mexicanos, 5: 2 (1989), pp. 313-326. 